\title{
Long-term cardiac composite risk following adjuvant treatment in breast cancer patients
}

\author{
Hong Bae Choi', Sangchul Yun', Sung Woo Cho', Min Hyuk Lee', Jihyoun Lee', Suyeon Park ${ }^{2}$ \\ 'Department of Surgery, Soonchunhyang University Seoul Hospital, Seoul; \\ ${ }^{2}$ Department of Biostatistics, Soonchunhyang University Seoul Hospital, Soonchunhyang University College of Medical Sciences, Seoul, Korea
}

Purpose: Cardiotoxicity is a serious late complication of breast cancer treatment. Individual treatment risk of specific drugs has been investigated. However, studies on the evaluation of the composite risk of chemotherapeutic agents are limited.

Methods: We retrospectively analyzed the medical records of breast cancer patients who received adjuvant treatment and had available serial echocardiography results. Patients were assigned to subgroups based on chemotherapy containing anthracyclines (A), anthracyclines and taxanes (A+T), and radiotherapy (RT). The development of cardiac disease and serial ejection fraction (EF) were reviewed. EF decline up to $10 \%$ from baseline was considered grade 1 cardiotoxicity and EF decline $>20 \%$ or absolute value $<50 \%$ was considered grade 2 cardiotoxicity. The most recent medical records and echocardiography results over 1 year of chemotherapy completion were also reviewed. Late cardiotoxicity was defined as a lack of recovery of EF decline or aggravated EF decline from baseline.

Results: In total, 123 patients were evaluated. A small reduction in EF was observed after chemotherapy in both chemotherapy groups. There were no significant differences between groups $A$ and $A+T$ in EF decline following chemotherapy. We could not find any differences in composite risk between the chemotherapy groups and the RT group during follow-up. Late cardiotoxicity was seen in $15.45 \%$ of patients. During follow-up, three patients were diagnosed with dilated cardiomyopathy.

Conclusion: There was no significant composite risk elevation following adjuvant treatment of breast cancer. However, late cardiotoxicity was considerable and further research in this direction is necessary.

Keywords: Breast neoplasms, Heart diseases, Chemotherapy, Adjuvant, Echocardiography

\section{INTRODUCTION}

Increased awareness of cancer screening and improved treatment options lead to increased breast cancer survival rate. The late effects of breast cancer treatment may constitute important concerns

Received: Jun 19, 2018 Revised: Sep 5, 2018 Accepted: Sep 13, 2018

Correspondence to: Jihyoun Lee

Department of Surgery, Soonchunhyang University Seoul Hospital,

59 Daesagwan-ro, Yongsan-gu, Seoul 04401, Korea

Tel: +82-2-709-9499, Fax: +82-2-709-9624

E-mail: Ihthof@hanmail.net

ORCID: Hong Bae Choi (https://orcid.org/0000-0002-6540-7309), Sangchul Yun (https://orcid.org/0000-0002-6321-4319), Sung Woo Cho (https://orcid.org/00000002-7206-798X), Min Hyuk Lee (https://orcid.org/0000-0001-9003-8819), Jihyoun Lee (https://orcid.org/0000-0003-0041-8717), Suyeon Park (https://orcid.org/00000002-6391-557X)

Copyright (C) 2018 Korean Society of Surgical Oncology

This is an Open Access article distributed under the terms of the Creative Commons Attribution Non-Commercial License (http://creativecommons.org/licenses/by-nc/4.0) which permits unrestricted non-commercial use, distribution, and reproduction in any medium, provided the original work is properly cited. for "disease-free" survivors after primary treatment of breast cancer. Therefore, breast cancer survivorship research should be conducted not only for the recurrence of primary disease but also for the early and late effects of primary treatment [1].

According to results of a recently reported breast cancer cohort study of long-term observation, $49.9 \%$ of deaths were caused by breast cancer and $16.3 \%$ were as a result of cardiac diseases [2]. Another study showed that for patients over the age of 70 , a higher proportion of deaths resulted from cardiac diseases than from breast cancer-related causes [3]. Furthermore, heart disease as a cause of death is frequently reported in breast cancer survivors with values higher than those for survivors of any other type of cancer [4].

The development of cardiac diseases [5] has been reported to be associated with chemotherapy, especially with anthracyclines [6,7], and also with cyclophosphamide, capecitabine, docetaxel, paclitaxel [8]. Old-fashioned radiotherapy and trastuzumab treatment have also been studied for cardiac risks [9]. Regarding endocrine treatment, cholesterol levels have been considered as related to 
heart disease development. Tamoxifen is known to decrease the risk of heart diseases. Additionally, it is thought that aromatase inhibitors might carry higher cardiac risk than tamoxifen [10]. In terms of cardiac risk, a head-to-head evaluation of individual regimens has been reported in clinical trials [11,12]. However, it only presents the results of chemotherapeutic regimen, additional chemotherapeutic agents or target agents and studies on the composite risk of treatments are lacking.

It is known that most cardiac events develop within 12 months of chemotherapy [13]. Clinical guidelines recommend performing echocardiography within 6 to 12 months after chemotherapy [14]. However, there is no recommendation of surveillance if there are no cardiac toxicities after this period of time.

In this study, we reviewed the changes of cardiac function following adjuvant treatment of breast cancer and compared the composite risk of therapeutic agents in the late period of survivorship.

\section{METHODS}

From January 2011 to December 2014, we retrospectively analyzed patients who were diagnosed with breast cancer and received adjuvant chemotherapy at a single tertiary medical center. Patients who had serial echocardiographic results and confirmed follow-ups until December 31, 2017 or verified death records were included. Echocardiographic results were considered as pre-chemotherapy when obtained within 1-month of chemotherapy and post-chemotherapy if performed within 1 year after chemotherapy. Late follow-up results were selected 1 year after chemotherapy, or as recent as possible. Preoperative history of hypertension, diabetes, and dyslipidemia were reviewed, along with previous heart disease. We excluded subjects who had been heavily treated for local recurrence or metastasis of breast cancer during the follow-up period.

To evaluate composite risk, we assigned patients into anthracycline-containing chemotherapy without taxane (A) group or anthracycline with taxane $(\mathrm{A}+\mathrm{T})$ group. During the follow-up, cases of additional administration of anthracyclines or taxanes adding to adjuvant treatment were removed from evaluation to prevent the effect of increased cumulative dose. Therefore, patients heavily treated with anthracycline with dexrazoxane were not included. We divided the patients into subgroups based on whether or not they received left-sided radiotherapy. We reviewed patient data such as age, presence of co-morbidities, menopausal state, type of surgery, chemotherapy and radiotherapy history, and pathologic information of breast cancer. We collected echocardiographic data of systolic and diastolic left ventricular diameters (LVDs and LVDd), and ejection fraction (EF). The degree of cardiotoxicity was considered as grade 1 if the EF decline was up to $10 \%$ from baseline, and grade 2 if the $\mathrm{EF}$ decline was up to $20 \%$ from baseline or if $\mathrm{EF}$ was $<50 \%$. We reviewed the electronic charts and observed the occurrence of heart diseases such as congestive heart failure (CHF) or myocardial infarction (MI), and cardiac deaths, as well as breast cancer-related deaths. We then compared the incidences of cardiotoxicity for a year after chemotherapy completion. Long-term composite risks were evaluated in instances of newly developed cardiac diseases, no improvement or aggravation of cardiac toxicities in late follow-up, or new cases of cardiac toxicity where there was no evidence at the time of chemotherapy.

We used SPSS version 21 (IBM Corp, Armonk, NY, USA) for statistical analysis. Mann-Whitney U-test was used to evaluate baseline characteristics between groups A and A+T. Long-term composite risk of adjuvant treatment adding radiotherapy evaluation was performed by Cox regression analysis. A P-value $<0.05$ was considered statistically significant. This study was approved by Institutional Review Board of Soonchunhyang University Seoul Hospital (IRB No. 2018-04-027-002). The informed consent was waived.

\section{RESULTS}

A total of 123 patients were evaluated. Of these, 58 had anthracycline-containing chemotherapy (A) and 65 had anthracycline- and taxane-containing chemotherapy $(\mathrm{A}+\mathrm{T})$. The mean cumulative dose of anthracycline was $300 \mathrm{mg} / \mathrm{m}^{2}$ in A group, and $257.5 \mathrm{mg} / \mathrm{m}^{2}$ in $\mathrm{A}+\mathrm{T}$ group. The age at diagnosis of breast cancer was $47.98 \pm$ 8.52 in group $A$ and $48.63 \pm 7.19$ in group A+T. In group A, 58 patients (47\%) received chemotherapy with 5-fluorouracil doxorubicin, cyclophosphamide (FAC) regimen, in group $\mathrm{A}+\mathrm{T}, 46$ patients (37\%) received AC-T, 16 patients (13\%) received docetaxel and doxorubicin (TA), and three patients (2.4\%) received chemotherapy with the docetaxel, doxorubicin, cyclophosphamide (TAC) regimen. Of these, 15 patients received neoadjuvant chemotherapy with TA. There were no statistically significant differences in age and menopausal status between the two subgroups (Table 1). The prevalence of co-morbidities at the time of breast cancer diagnosis was evenly distributed among patients with diabetes $(\mathrm{P}=0.34)$, hypertension ( $\mathrm{P}=0.52)$, and dyslipidemia $(\mathrm{P}=0.10)$. More than $90 \%$ of the patients (58 patients in A group, 58 patients in $\mathrm{A}+\mathrm{T}$ group) had $\mathrm{T} 1$ and $\mathrm{T} 2$ tumors and did not show significant differences in T stage. However, $84.5 \%$ (49 patients) of patients in group A had $\mathrm{N} 0$ stage while $90.5 \%$ (57 patients) of patients in group A+T showed higher than N1 status. Most of the tumors were of the invasive ductal carcinoma histologic type. Breast-conserving surgery was the most common choice in approximately half of the cases in both groups (50.0\% [29 patients] in group A and 46.2\% [30 pa- 
Table 1. Baseline characteristics and type of adjuvant treatment

\begin{tabular}{|c|c|c|c|}
\hline Characteristic & $\begin{array}{l}\text { Anthracycline- } \\
\text { containing } \\
\text { (FAC) }\end{array}$ & $\begin{array}{l}\text { Anthracycline } \\
\text { and taxane }\end{array}$ & P-value \\
\hline Total & $58(100)$ & 65 (100) & \\
\hline Age (yr) & $47.98 \pm 8.52$ & $48.63 \pm 7.19$ & 0.26 \\
\hline $\mathrm{BMI}\left(\mathrm{kg} / \mathrm{m}^{2}\right)$ & $23.29 \pm 2.85$ & $23.34 \pm 3.65$ & 0.51 \\
\hline Smoking & 0 & $2(3.1)$ & 0.18 \\
\hline \multicolumn{4}{|l|}{ Co-morbidities } \\
\hline Diabetes & $3(5.2)$ & $1(1.5)$ & 0.34 \\
\hline Hypertension & $11(19)$ & $16(24.6)$ & 0.52 \\
\hline Dyslipidemia & $3(5.2)$ & 0 & 0.10 \\
\hline Menopausal status & & & 1.00 \\
\hline Premenopausal & $33(56.9)$ & 37 (56.9) & \\
\hline Postmenopausal & 25 (43.1) & $28(43.1)$ & \\
\hline T stage & & & 0.17 \\
\hline $\mathrm{T} 1$ & $27(46.6)$ & $24(38.1)$ & \\
\hline $\mathrm{T} 2$ & $31(53.4)$ & $34(54.0)$ & \\
\hline T3 & 0 & $4(6.3)$ & \\
\hline $\mathrm{T} 4$ & 0 & $1(1.6)$ & \\
\hline N stage & & & $<0.01$ \\
\hline NO & $49(84.5)$ & $6(9.5)$ & \\
\hline N1 & $7(12.1)$ & $41(65.1)$ & \\
\hline N2 & $1(1.7)$ & $12(19.0)$ & \\
\hline N3 & $1(1.7)$ & $4(6.3)$ & \\
\hline Histology & & & 0.41 \\
\hline Ductal & $55(94.8)$ & 59 (90.8) & \\
\hline Lobular & $1(1.7)$ & $4(6.2)$ & \\
\hline Others & $2(3.4)$ & $2(3.1)$ & \\
\hline Hormone receptor & & & 0.001 \\
\hline ER and/or PR positive & $27(46.6)$ & $49(75.4)$ & \\
\hline ER and/or PR negative & $31(53.4)$ & $16(24.6)$ & \\
\hline HER2 & & & 0.75 \\
\hline Negative & $33(56.9)$ & $47(72.3)$ & \\
\hline Positive & $25(43.1)$ & $18(27.7)$ & \\
\hline Surgery & & & 0.94 \\
\hline Breast-conserving surgery & $29(50.0)$ & 30 (46.2) & \\
\hline Mastectomy & $14(24.1)$ & $14(21.5)$ & \\
\hline SSM or NSM & $15(25.9)$ & 19 (29.2) & \\
\hline No surgery & 0 & $2(1.6)$ & \\
\hline Endocrine treatment & & & 0.001 \\
\hline No & $32(55.2)$ & $17(26.2)$ & \\
\hline Tamoxifen & $18(31.0)$ & $28(43.1)$ & \\
\hline$A l^{a)}$ & $7(12.1)$ & $18(27.7)$ & \\
\hline Tamoxifen followed by Al & $1(1.7)$ & $1(1.5)$ & \\
\hline OFS with tamoxifen & 0 & $1(1.5)$ & \\
\hline Radiotherapy & & & 0.22 \\
\hline No & $31(53.4)$ & $29(44.6)$ & \\
\hline Left sided & $16(27.6)$ & $17(26.2)$ & \\
\hline Right sided & $11(19.0)$ & $19(29.2)$ & \\
\hline Trastuzumab & & & 0.23 \\
\hline No & $43(74.1)$ & $54(83.1)$ & \\
\hline Yes & $15(25.9)$ & $11(16.9)$ & \\
\hline
\end{tabular}

Values are presented as number (\%) or mean \pm SD.

FAC, 5-fluorouracil, doxorubicin, cyclophosphamide; BMI, body mass index; $E R$, estrogen receptor; $P R$, progesterone receptor; HER2, human epidermal growth factor receptor 2; SSM, skin sparing mastectomy; NSM, nipple sparing mastectomy; $\mathrm{Al}_{1}$ aromatase inhibitor; OFS, ovarian function suppression.

a) Letrozole, anastrozole, and exemestane are included.

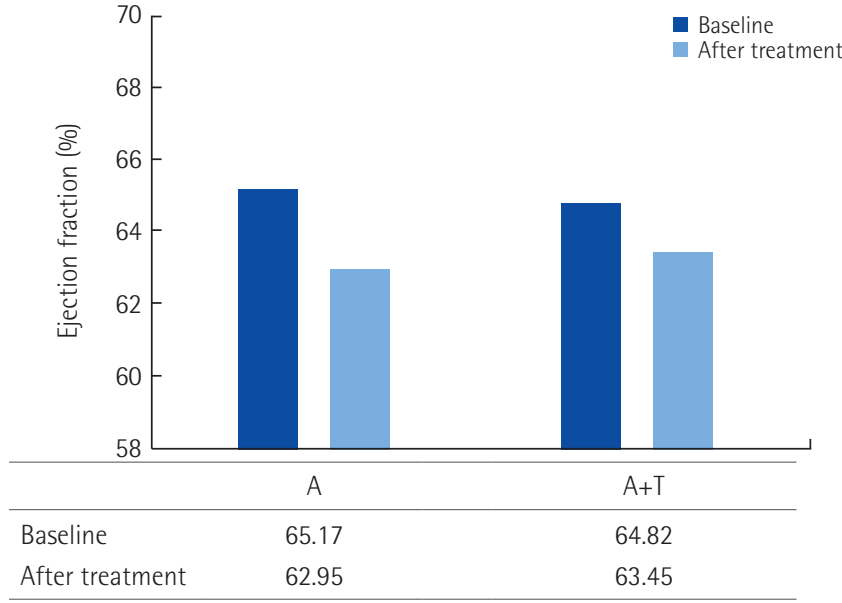

$A$, anthracycline-containing regimen without radiotherapy (RT); $A+T$, anthracycline and taxane without $R T$.

Fig. 1. Influence of treatment composite to ejection fraction. It was measured baseline and after treatment combination. It shows comparison of ejection fraction by anthracycline containing treatment and anthracycline and taxane within 1 year after chemotherapy.

tients] in group $\mathrm{A}+\mathrm{T})$ and there were no significant differences in both groups. In group A, 28 patients received radiotherapy and $27.6 \%$ of them were left-sided. In group A+T, 36 patients received radiotherapy with $26.2 \%$ (17 patients) of them left-sided. There were no significant differences in human epidermal growth factor receptor 2 status for both groups and in the proportion of patients who received trastuzumab therapy. We compared left ventricular ejection fractions before and after chemotherapy using echocardiography. EF reductions after chemotherapy were lower for both chemotherapy groups, but the value was small and not statistically significant (Fig. 1).

The total person-year observed was 523.79 years. The mean follow-up period was 4.26 years ( $3.37-5.55$ years). During the follow-up, two patients died from breast cancer and three patients were newly diagnosed with dilated cardiomyopathy (DCMP). All of them were in their 50s and did not receive left-sided radiotherapy. One of them received anthracycline and the others received anthracycline and taxane treatment. We evaluated cardiotoxicities including EF decline with person-time adjustment. A total of 19 patients (15.45\%) presented with late cardiotoxicity. There was no difference in the follow-up length between the groups (Table 2). Incidence of observed cardiotoxicities within 1 year after chemotherapy was similar between groups $\mathrm{A}$ and $\mathrm{A}+\mathrm{T}$ (hazard ratio [HR], 1.06; 95\% confidence interval [CI], 0.43-2.61; $\mathrm{P}=0.90$ ). There were no significant differences in long-term composite cardiac risk between subgroups adding anthracyclines, taxane, and left-sided radiotherapy (HR, 1.63; 95\% CI, 0.39-6.93). And also 
Table 2. Comparison of long-term composite risk of adjuvant treatment of breast cancer

\begin{tabular}{|c|c|c|c|c|c|c|}
\hline Subgroup & $\begin{array}{c}\text { No. of } \\
\text { patients }\end{array}$ & $\begin{array}{l}\text { Person-year } \\
\text { Mean (Cl) }\end{array}$ & P-value & $\begin{array}{l}\text { Cardiotoxicity } \\
(\%)^{\mathrm{a})}\end{array}$ & HR $(95 \% \mathrm{Cl})$ & P-value \\
\hline \multicolumn{7}{|l|}{ Chemotherapy regimen } \\
\hline Anthracycline-containing (FAC) & 58 & $6.12(5.60-6.64)$ & 0.90 & $9(15.52)$ & 1 & 0.90 \\
\hline Anthracycline and taxane & 65 & $6.07(5.57-6.57)$ & & $10(15.38)$ & $1.06(0.43-2.62)$ & \\
\hline \multicolumn{7}{|c|}{ Chemotherapy regimen adding radiotherapy } \\
\hline A & 42 & $6.30(5.60-6.85)$ & 0.70 & $5(11.90)$ & 1 & \\
\hline$A+R T$ & 16 & $5.60(4.44-6.77)$ & & $4(25.0)$ & $2.12(0.57-7.92)$ & 0.26 \\
\hline$A+T$ & 48 & $6.14(5.59-6.70)$ & & $7(14.58)$ & $1.30(0.41-4.10)$ & 0.65 \\
\hline$A+T+R T$ & 17 & $5.30(4.52-6.09)$ & & $3(17.64)$ & $1.63(0.39-6.93)$ & 0.51 \\
\hline
\end{tabular}

$\mathrm{Cl}$, confidence interval; $\mathrm{HR}$, hazard ratio; FAC, 5-fluorouracil, doxorubicin, cyclophosphamide; RT, radiotherapy; $\mathrm{A}$, anthracycline-containing regimen without RT; $A+R T$, anthracycline and $R T ; A+T$, anthracycline and taxane without $R T ; A+T+R T$, anthracycline, taxane, and $R T$.

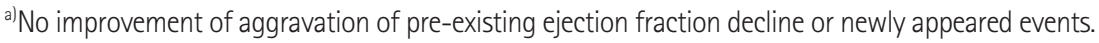

Table 3. Cardiac outcome measures by treatment

\begin{tabular}{|c|c|c|c|c|c|c|c|c|c|}
\hline \multirow[b]{2}{*}{ Subgroup } & \multicolumn{3}{|c|}{ LVDs (mm) } & \multicolumn{3}{|c|}{$\operatorname{LVDd}(\mathrm{mm})$} & \multicolumn{3}{|c|}{ LVEF (\%) } \\
\hline & Baseline & $\begin{array}{c}\text { After } \\
\text { treatment }\end{array}$ & P-value & Baseline & $\begin{array}{c}\text { After } \\
\text { treatment }\end{array}$ & P-value & Baseline & $\begin{array}{c}\text { After } \\
\text { treatment }\end{array}$ & P-value \\
\hline Anthracycline-containing (FAC) & $28.03 \pm 3.24$ & $29.59 \pm 3.48$ & $<0.001$ & $46.50 \pm 3.51$ & $47.26 \pm 4.26$ & 0.185 & $65.17 \pm 4.87$ & $62.95 \pm 6.24$ & 0.023 \\
\hline Anthracycline and taxane & $27.84 \pm 3.46$ & $28.22 \pm 3.75$ & 0.479 & $45.95 \pm 3.69$ & $45.48 \pm 4.02$ & 0.427 & $64.82 \pm 6.07$ & $63.45 \pm 4.89$ & 0.136 \\
\hline
\end{tabular}

Values are presented as mean \pm SD.

LVDs, left ventricular diameter, systolic; LVDd, left ventricular diameter, diastolic; LVEF, left ventricular ejection fraction; FAC, 5-fluorouracil, doxorubicin, cyclophosphamide regimen.

there were no statistical differences in the left ventricular diameter and ejection fraction between the $\mathrm{A}$ group and the $\mathrm{A}+\mathrm{T}$ group before and after treatment (Table 3). A total of 26 patients (15 in A group, 11 in the $\mathrm{A}+\mathrm{T}$ group) were treated with Herceptin in the chemotherapy group, of which six patients had cardiotoxicity (three in the A group, three in the A+T group).

Most of the patients of long-term cardiac toxicity were asymptomatic during the time of retrospective observation, therefore routine echocardiography follow-up was conducted for those patients. One patient from A group (1.72\%) and one patient from $\mathrm{A}+\mathrm{T}$ group (1.54\%) was received medical treatment later for management of hypertension

\section{DISCUSSION}

In this study, we found that late cardiotoxicity, defined as a lack of recovery or aggravation of $\mathrm{EF}$ decline or newly diagnosed cardiac disease, persisted for long periods. There were no significant additional composite risks from adding taxanes and left-sided radiotherapy to adjuvant anthracyclines. There was a low incidence of DCMP development, however, considerable persistent cardiotoxicity was seen during the long-term observation.

It is well-known that chemotherapy with anthracyclines carries a risk to cardiac function [15]. At first notice of signs of EF decline, it is imperative that management is prompt because beneficial treatment response is uncertain 6 months after EF decline onset [16]. There are no definite biomarkers for early detection. However, recently the use of troponin I or pro-brain natriuretic peptide has been investigated. There is currently no optimal timing or cutoff level for the use of biomarkers; therefore, the routine use of cardiac biomarkers in asymptomatic patients is not recommended [17]. Although we could not find additional risks of adjuvant treatment, we noted that its effect is sustained until the late period of survivorship. If the echocardiography is not affordable, it might be helpful to have a reliable cardiac biomarker that can be readily tested. In addition, management of other risk factors that can cause cardiac diseases might be important. Besides tight control of blood glucose, lipid profile, and blood pressure, the development of cardiac disease is also strongly related to obesity and exercise. Waist circumference is associated with elevated risk [18]. Exercise guideline adherence is also related to reduction of cardiovascular risks [19].

Before the introduction of novel techniques in the 1980's, radiation therapy, whether left or right-sided, was a significant risk factor for the development of cardiac diseases such as MI, CHF, and valvular disease. Long-term survivors who received radiotherapy 
before 1980 showed increased risk of MI and CHF [20]. Receiving adjuvant chemotherapy and smoking were the risk factors for cardiac complications. Recently, many modern techniques to minimize dose delivery to the thorax have been reported. According to these reports, these modern techniques may carry less cardiac risks [21], which is consistent with the findings of this study. We selected left-sided radiotherapy so as to evaluate the effects of accidental dose delivery to the heart, but we did not find any relationship between long-term risk of cardiac complications and left-sided radiotherapy.

In a study conducted in a long-term survivor cohort and cancer-free controls, there was no difference in newly diagnosed diseases [22] in over 10 years of follow-up. There were even slight shifts to the left for circulatory diseases. This finding should be further investigated as it contradicts the traditional view that adjuvant therapy may carry a long-term risk of cardiac disease. The limitation of this study is that it was a single-center review and included a small population sample. More research is needed to evaluate the real-world composite risk of adjuvant treatment in a large population sample to effectively settle conflicting views with reproducible evidence. In addition, we could not evaluate the effects of trastuzumab or endocrine treatment, which may also be important composite risk factors, because of the small population sample studied.

Although this study showed no additional composite risks of cardiotoxic adjuvant therapies, cardiac complication is still a significant threat to the health of breast cancer survivors. And the mean cumulative dose of anthracycline was lower in $\mathrm{A}+\mathrm{T}$ group, which may affect the incidence of cardiac toxicity. Further evaluation in a large cohort with comparative study is required to provide evidence from surveillance for cardiac disease development in long-term breast cancer survivors.

\section{CONFLICT OF INTEREST}

No potential conflict of interest relevant to this article was reported.

\section{ACKNOWLEDGMENTS}

This research is supported by the Soonchunhyang University Research Fund.

\section{REFERENCES}

1. Wilbur J. Surveillance of the adult cancer survivor. Am Fam Physician 2015;91:29-36.

2. Abdel-Qadir H, Austin PC, Lee DS, Amir E, Tu JV, Thavendirana- than P, et al. A Population-based study of cardiovascular mortality following early-stage breast cancer. JAMA Cardiol 2017;2:88-93.

3. Park NJ, Chang Y, Bender C, Conley Y, Chlebowski RT, van Londen GJ, et al. Cardiovascular disease and mortality after breast cancer in postmenopausal women: results from the Women's Health Initiative. PLoS One 2017;12:e0184174.

4. Stava C, Weiss LT, Vassilopoulou-Sellin R. Health profiles of 814 very long-term breast cancer survivors. Clin Breast Cancer 2006;7: 228-36.

5. Zagar TM, Cardinale DM, Marks LB. Breast cancer therapy-associated cardiovascular disease. Nat Rev Clin Oncol 2016;13:172-84.

6. Guo S, Wong S. Cardiovascular toxicities from systemic breast cancer therapy. Front Oncol 2014;4:346.

7. Mulrooney DA, Yeazel MW, Kawashima T, Mertens AC, Mitby P, Stovall M, et al. Cardiac outcomes in a cohort of adult survivors of childhood and adolescent cancer: retrospective analysis of the Childhood Cancer Survivor Study cohort. BMJ 2009;339:b4606.

8. Chang HM, Okwuosa TM, Scarabelli T, Moudgil R, Yeh ET. Cardiovascular complications of cancer therapy: best practices in diagnosis, prevention, and management: Part 2. J Am Coll Cardiol 2017; 70:2552-65.

9. Herrmann J, Yang EH, Iliescu CA, Cilingiroglu M, Charitakis K, Hakeem A, et al. vascular toxicities of cancer therapies: the old and the new: an evolving avenue. Circulation 2016;133:1272-89.

10. Haque R, Shi J, Schottinger JE, Chung J, Avila C, Amundsen B, et al. Cardiovascular disease after aromatase inhibitor use. JAMA Oncol 2016;2:1590-7.

11. Hatam N, Ahmadloo N, Ahmad Kia Daliri A, Bastani P, Askarian M. Quality of life and toxicity in breast cancer patients using adjuvant TAC (docetaxel, doxorubicin, cyclophosphamide), in comparison with FAC (doxorubicin, cyclophosphamide, 5-fluorouracil). Arch Gynecol Obstet 2011;284:215-20.

12. Martin M, Lluch A, Segui MA, Ruiz A, Ramos M, Adrover E, et al. Toxicity and health-related quality of life in breast cancer patients receiving adjuvant docetaxel, doxorubicin, cyclophosphamide (TAC) or 5-fluorouracil, doxorubicin and cyclophosphamide (FAC): impact of adding primary prophylactic granulocyte-colony stimulating factor to the TAC regimen. Ann Oncol 2006;17:120512 .

13. Cardinale D, Colombo A, Bacchiani G, Tedeschi I, Meroni CA, Veglia F, et al. Early detection of anthracycline cardiotoxicity and improvement with heart failure therapy. Circulation 2015;131:1981-8.

14. Armenian SH, Lacchetti C, Barac A, Carver J, Constine LS, Denduluri $\mathrm{N}$, et al. Prevention and monitoring of cardiac dysfunction in survivors of adult cancers: American Society of Clinical Oncology clinical practice guideline. J Clin Oncol 2017;35:893-911.

15. Domercant J, Polin N, Jahangir E. Cardio-oncology: a focused re- 
view of anthracycline-, human epidermal growth factor receptor 2 inhibitor-, and radiation-induced cardiotoxicity and management. Ochsner J 2016;16:250-6.

16. Cardinale D, Colombo A, Lamantia G, Colombo N, Civelli M, De Giacomi G, et al. Anthracycline-induced cardiomyopathy: clinical relevance and response to pharmacologic therapy. J Am Coll Cardiol 2010;55:213-20.

17. Denlinger CS, Sanft T, Baker KS, Baxi S, Broderick G, Demark-Wahnefried W, et al. Survivorship, version 2.2017, NCCN clinical practice guidelines in oncology. J Natl Compr Canc Netw 2017;15:1140-63.

18. Cespedes Feliciano EM, Kwan ML, Kushi LH, Weltzien EK, Castillo AL, Caan BJ. Adiposity, post-diagnosis weight change, and risk of cardiovascular events among early-stage breast cancer survivors.
Breast Cancer Res Treat 2017;162:549-57.

19. Scott JM, Adams SC, Koelwyn GJ, Jones LW. Cardiovascular Late effects and exercise treatment in breast cancer: current evidence and future directions. Can J Cardiol 2016;32:881-90.

20. Hooning MJ, Botma A, Aleman BM, Baaijens MH, Bartelink H, Klijn JG, et al. Long-term risk of cardiovascular disease in 10-year survivors of breast cancer. J Natl Cancer Inst 2007;99:365-75.

21. Leung HW, Chan AL, Muo CH. Late cardiac morbidity of adjuvant radiotherapy for early breast cancer: a population-based study. JCardiol 2016;67:567-71.

22. Lash TL, Thwin SS, Yood MU, Geiger AM, Bosco J, Quinn VP, et al. Comprehensive evaluation of the incidence of late effects in 5-year survivors of breast cancer. Breast Cancer Res Treat 2014;144:643-63. 